\title{
FGF21 - the cause of having a 'sweet tooth'?
}

Fibroblast growth factor 21 (FGF21), a hepatokine, has been implicated in preference for sweet foods in humans for the first time.

Previous work in rodents had shown that FGF21 secreted from the liver regulated the intake of sweet food. In addition, findings suggested that FGF21 suppressed the 'sweet tooth' in primates. "Yet it was not clear that the hormone would play the same role in humans," say Matthew Gillum and Niels Grarup (co-senior authors).

Using a combination of a genetic epidemiology study and a clinical study, Gillum, Grarup and colleagues aimed to determine whether FGF21 was involved in the intake of sweet foods in humans. In the first part of the work, the investigators genotyped variants in the FGF21 locus in more than 6,000 participants in the Inter99 cohort in Denmark. Participants also provided detailed information on their diet using a 198-item food frequency questionnaire. The researchers generated frequency scores to summarize the weekly intake of sweet snacks between meals, grouping them into a candy (or sweets) category and a cake category.

Carriers of the rs 838133

A allele of FGF21 had an increased odds ratio of being in the highest tertile for intake of all types of sweet-tasting foods. When analysed by category, candy intake was higher in the A-allele group, but cake intake was similar between the groups. "Individuals with particular variants of the FGF21 gene were about $20 \%$ more likely to be top ranking consumers of sweets and candy," explain Gillum and Grarup.

The clinical study included 86 participants who completed a questionnaire to determine their preferences for sweet, fatty sweet and salty foods. The analysis focused on the 25 participants from the highest tertile of sweet preference ('sweet-likers') and the 26 participants from the lowest tertile of sweet preference ('sweet-dislikers'). The researchers measured levels of FGF21 in these participants following a $12 \mathrm{~h}$ fast and found that levels were $51 \%$ higher in sweet-dislikers than in sweet-likers. In addition, a sucrose challenge after a $12 \mathrm{~h}$ fast demonstrated that levels of FGF21 increase after sucrose consumption in both sweet-likers and sweet-dislikers. "We thus implicate the FGF21 hormone in the regulation of food preference and sugar intake in humans and show that at least a small part of the individual propensity

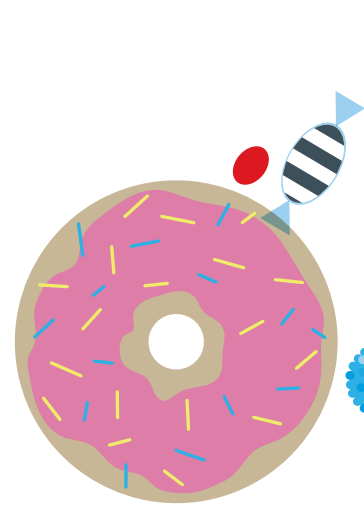

to sweet snacking is determined by genetic variation of the FGF21 gene," conclude Gillum and Grarup.

The researchers note that more work needs to be done to fully understand the roles of FGF21 in humans. Gillum, Grarup and colleagues are particularly interested in conducting larger human studies to more precisely determine how circulating levels of FGF21 relate to food preference and intake, as well as cardiometabolic disorders such as obesity and type 2 diabetes mellitus. "We would also like to conduct a clinical trial in humans to study the effect of FGF21 administration on sweet preference and body weight," say Gillum and Grarup. "It would also be extremely interesting to further elucidate the mechanism behind the sweet tooth in humans, for instance by using genetic studies to discover novel factors."

Claire Greenhill

ORIGINAL ARTICLE Søberg, S. et al. FGF21 is

a sugar-induced hormone associated with sweet intake and preference in humans. Cell Metab. http://dx.doi.org/10.1016/j.cmet.2017.04.009 (2017)

Individuals
with particular
variants of the
FGF21 gene
were about
$20 \%$ more
likely to be
top ranking
consumers of
sweets and
candy

